\title{
Adaptive Hidden Markov Model With Anomaly States for Price Manipulation Detection
}

\author{
Yi Cao, Yuhua Li, Senior Member, IEEE, Sonya Coleman, Member, IEEE, Ammar Belatreche, \\ and Thomas Martin McGinnity, Senior Member, IEEE
}

\begin{abstract}
Price manipulation refers to the activities of those traders who use carefully designed trading behaviors to manually push up or down the underlying equity prices for making profits. With increasing volumes and frequency of trading, price manipulation can be extremely damaging to the proper functioning and integrity of capital markets. The existing literature focuses on either empirical studies of market abuse cases or analysis of particular manipulation types based on certain assumptions. Effective approaches for analyzing and detecting price manipulation in real time are yet to be developed. This paper proposes a novel approach, called adaptive hidden Markov model with anomaly states (AHMMAS) for modeling and detecting price manipulation activities. Together with wavelet transformations and gradients as the feature extraction methods, the AHMMAS model caters to price manipulation detection and basic manipulation type recognition. The evaluation experiments conducted on seven stock tick data from NASDAQ and the London Stock Exchange and 10 simulated stock prices by stochastic differential equation show that the proposed AHMMAS model can effectively detect price manipulation patterns and outperforms the selected benchmark models.
\end{abstract}

Index Terms-Anomaly detection, capital market microstructure, feature extraction, hidden Markov model (HMM), market abuse, price manipulation.

\section{INTRODUCTION}

$\mathbf{R}^{1}$ ISK management has been a major concern for banks, portfolio managers, and companies concerned with stock exchange transactions for many years. However, new aspects of risk management have been identified by regulators after the financial crisis in 2008 and especially since the flash crash in 2010. One important aspect is the surveillance of financial exchange market for preventing market abuse activities. The abuse of financial markets can take on a variety of forms, all of which can be extremely damaging to the proper functioning and integrity of the market. The forms contain three primary categories, namely information-based

Manuscript received July 1, 2013; revised March 25, 2014; accepted March 28, 2014. Date of publication April 22, 2014; date of current version January 15,2015 . This work was supported by the companies and organizations involved in the Northern Ireland Capital Markets Engineering Research Initiative.

Y. Cao, Y. Li, S. Coleman, and A. Belatreche are with the Intelligent Systems Research Centre, University of Ulster, Londonderry BT48 7JL, U.K. (e-mail: cao-y1@email.ulster.ac.uk; y.li@ulster.ac.uk; sa.coleman@ulster.ac.uk; a.belatreche@ulster.ac.uk).

T. M. McGinnity is with the Intelligent Systems Research Centre, University of Ulster, Londonderry BT48 7JL, U.K., and also with the School of Science and Technology, Nottingham Trent University, Nottingham NG1 4BU, U.K. (e-mail: martin.mcginnity@ntu.ac.uk).

Color versions of one or more of the figures in this paper are available online at http://ieeexplore.iee.org.

Digital Object Identifier 10.1109/TNNLS.2014.2315042 manipulation, where false information (financial rumor) is released to affect the equity price, action-based manipulation, where the equity price is changed by actions of squeezing the supply/demand of the equity, and trade-based manipulation, where the equity price is shifted only by simply buying and selling [1]. A major format of the trade-based abuse is price manipulation, where the manipulation tactic targets the equity bid/ask prices only [1], [2].

Compared with the information and action-based manipulations, trade-based manipulation usually does not contain any illegitimate actions such as financial rumor spreading and market resource squeezing but is carried out only by legal trading activities. With carefully designed selling and buying sequences, manipulators could make the market bid/ask price change following their expectation. In the price manipulation tactics, a series of trading actions are often linked in contextual relationships. The monitoring of any single action would not contribute to a thorough detection of the underlying problem. This is the main challenge of such price manipulation detection algorithms. Another challenge arises from the fact that the manipulation behaviors are often not obvious when mixed with a mass of normal trading records. The evolution of manipulation strategies over time is also a challenge for the detection algorithms. Most of the existing related literature empirically studies the manipulations and the corresponding market responses. A very few works analyze the features of different price manipulation strategies and the corresponding detection approaches.

A number of pioneering papers introduced in [3], such as trading the foreign exchange by the neuro-wavelet hybrid system [4] and forecasting the bond price by least-square support vector machine [5], opened the door for more advances in modeling and predicting financial markets using computationally intelligent techniques. These received fresh attention after the 2007-2009 financial crisis recede. More accurate results than the traditional models were reported [6], [7]. Inspired by findings in those literatures, we proposed an approach that considers the challenges of the price manipulation detection problem. In this approach, we formulate the problem based on the analysis of typical cases, extract the intrinsic features of the manipulation patterns, and present a new model to detect price manipulation activities. The main contributions of our work are as follows.

The problem of price manipulation is discussed together with the analysis of three typical examples, from which the key feature patterns are extracted. This provides a clear problem formulation and explains the significance of exploring the 
price manipulation patterns. A hidden Markov model with anomaly states (HMMAS) is proposed to model and detect the price manipulation patterns. The HMMAS makes a detection decision based on a sequence of price information rather than a single value at a given time instant. The price manipulation types are also quantified and designated by the HMMAS based on the features of each manipulation type. To the best of our knowledge, this is a novel application of traditional hidden Markov model (HMM) customized to solving market abuse detection. To enhance the adaptability of the HMMAS, a traditional retraining mechanism is proposed for automatically tracking the changes in the statistical properties of the financial time series. Substantial experiments have been conducted on both real data from primary USA and U.K. markets for testing the practicability on real life and simulated data for testing the robustness to nonstationarity. This verification mechanism provides a complete spectrum of workability testing from both business and theoretic perspectives.

The remainder of this paper is organized as follows. Section II provides a brief review of price manipulation and the corresponding detection methods as well as the analysis of the manipulation cases and the formulated detection logic. The features of basic price manipulation cases are characterized and extracted by the proposed approaches in Section III. Section IV presents the proposed AHMMAS approach for detecting patterns of price manipulation. Performance evaluation of the proposed approach is provided in Section V. Finally, Section VI concludes this paper and discusses potential improvements and future work.

\section{Price Manipulation and Its Detection}

\section{A. Price Manipulation}

In capital markets, limit orders, the instructions to buy or sell equities, are submitted by investors to the electronic trading platform of one exchange market. The limit orders indicate the trading intention of the investor to buy or sell volumes of a specific equity at a specific price. The trading occurs once there are eligible matched orders from the investors. Among those outstanding unmatched limit orders, the highest price investors are willing to pay for an equity determines the bid price and the lowest price at which investors are willing to sell an equity decides the ask price. The gap between the bid and ask price is defined as the bid-ask spread [8].

Price manipulation can be carried out in many different forms [9]. One primary form is termed ramping (or gouging [10] and momentum ignition [11]), where one investor enters a buy/sell order, usually called a spoofing order at a price which is higher/lower than the bid/ask to create the appearance of active interest in a security, followed by a bona fide order on the opposite side of the order book waiting to be executed, subsequently followed by the withdrawal of the first order when the bona fide order was mostly executed. Once the spoofing order is created, a price upward/downward movement is expected from the manipulator and latter actions are followed only when there is an enough potential profit for the investor. Ramping has roughly the same effect as another two forms of price manipulation, pump and dump and capping

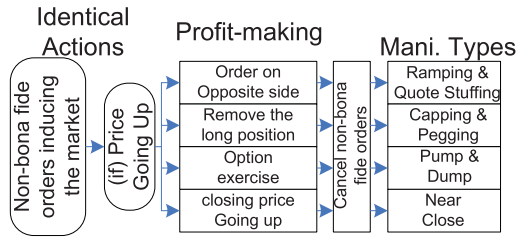

Fig. 1. Price manipulation.

TABLE I

Price Manipulation

\begin{tabular}{c|l}
\hline \hline Mani. Types & Typical Scenarios \\
\hline $\begin{array}{c}\text { ramping or Gouging } \\
\text { or quote stuffing } \\
\text { or capping \& pegging }\end{array}$ & $\begin{array}{l}\text { liquid stocks price manipulation } \\
\text { and the profit from opposite } \\
\text { orders or positions }\end{array}$ \\
\hline pump \& dump & $\begin{array}{l}\text { manipulation cross stock } \\
\text { or option markets. }\end{array}$ \\
\hline near close & market closing price manipulation. \\
\hline \hline
\end{tabular}

and pegging schemes [10], where the profit making approaches are different. In pump and dump, the exchange participants make profit by carrying out a quick flip at the manipulated price to remove the already held long positions (holding of shares for a stock is said to be has a long position in the stock). In capping and pegging, the held derivative (e.g., call option, defined as an agreement that gives an investor the right to buy a stock at a specific price within a specific time period) of the manipulated security is exercised if a price increase is generated. If the profit is exploited in another market, the form of price manipulation is then termed as cross order/market. If the closing price is manipulated, the manipulation is termed as near close.

In September 2012, an analogous type of ramping was reported and documented by the Financial Industry Regulatory Authority of the USA [12]. In this case, a liquidity-enhancing strategy, quote stuffing, was used for creating the fictitious wild impression of the buying interest on the bid side. As a result of the quote stuffing, where a mass of nonbona fide orders was submitted, the best bid price was pushed up $6.9 \mathrm{bps}^{1}$ and hence the bid-ask spread was narrowed by $87.5 \%$. The manipulators have benefited from the trading on their previous submitted order on the ask side at the pushed price. The manipulation process lasted for only $819 \mathrm{~ms}$ and the cancellation time of the nonbona fide orders was only $767 \mathrm{~ms}$.

Quote stuffing is one of the dominant manipulation strategies in a number of major exchange markets in Europe and USA [11], [13]. It floods the market with massive numbers of new orders, which are then cancelled in rapid successions for creating a large number of successive new best bidlask quoting, each potentially acting as a bait of an opposite order, upon which the potential profit might be realized. The only difference between ramping and quote stuffing is that the latter sweeps the order book with more spoofing orders and faster cancellation time.

As a summary, all of the above-mentioned forms of price manipulation refer to the same tactic: submitting nonbona fide orders to the market, taking advantage of the shifted prices

\footnotetext{
${ }^{1} \mathrm{~A}$ basis point is a unit equal to one hundredth of a percentage point.
} 
(if expected changes occurred), where the profits are made by distinct ways in various profit-making scenarios, as shown in Fig. 1 and Table I.

\section{B. Price Manipulation Detection}

The detection of price manipulation has however, been less studied in contrast to the volume of theoretical and empirical work on the manipulation activities. Two computational approaches for detecting trade-based manipulation within the emerging Istanbul Stock Market, logistic regression with an artificial neural network, and support vector machine, have been studied and compared [14]. The detection was based on empirical studies of the statistical features of daily return, daily trading volume, and daily volatilities. Higher deviations from the nonmanipulated cases indicated manipulation. Similar work has been done by first studying the manipulated case pursued by the Securities and Exchange Commission (SEC) and constructing a manipulated cases data set, then modeling the returns, liquidity, and volatility as well as the news and events related to the stocks during the manipulation period by linear and logistic regression [15]. Evaluations and comparisons of different techniques were presented in [14] and [15], yet both studies lack reliable quantitative analysis of the relationship between the manipulation tactic and the relevant features such as return, trading volume, liquidity, and volatility, which in most of the cases are the result of economic cycles, market (index) moves, and even public events. The detection models based on the significant deviation of those market attributes are doomed to suffer from the error rate of the unusual but legitimate activities that are recognized as manipulation [14], [15]. Therefore, there is a knowledge gap between the data features and the detection techniques.

A very simple technique, association rule, has been proposed for detecting the closing price manipulation in the Thai bond market [16]. The approach was based on the assumption that the trading time of a trader should be random regardless of the traders. Therefore, any association between a trader and the transaction orders may indicate price manipulation. This approach has been stated as a supplementary to the existing surveillance system within the Thai bond market for identifying a specific type of trade-based manipulation [16] rather than a general detection approach.

A market close ramping detection algorithm developed by Smarts Group International, a surveillance technique provider, and applied in NASDAQ OMX for assisting regulators and brokers in detecting trade-based manipulation was discussed in [17]. This paper mainly focused on the empirical study of relationships between the market efficiency and the manipulations detected by the algorithm from Smarts Group rather than the analysis of detection algorithms. The algorithm detected market close ramping according to the historical price change where the threshold was set as the $99 \%$ histogram distribution cutoff of the historical price change during the benchmark period. A market close ramping alert was triggered if the changes of the closing price and the price $15 \mathrm{~min}$ prior were greater than the chosen threshold [17]. Though straightforward, the market proven detection approach from
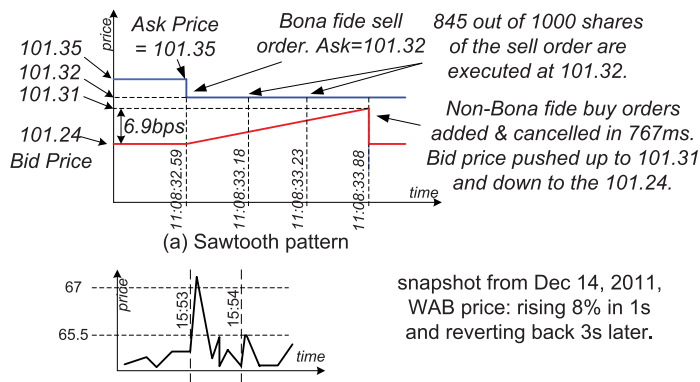

(b) Square pattern

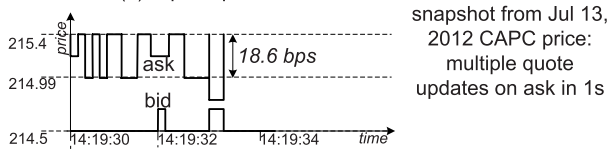

(c) Pulse pattern

Fig. 2. Feature extraction module. (a) Sawtooth pattern. (b) Square pattern. (c) Pulse pattern.

Smarts Group International provided an industry reference for the study presented in this paper.

To date, research has mainly focused on the detection of manipulation in prerecorded data sets according to the assumptions from empirical studies. To the best of our knowledge, only limited efforts appear to have been made in deep analysis of the strategic behavior of price manipulation tactic [18] and to a lesser extent on computational modeling of the manipulation strategies [17], which is the focus of this paper.

\section{Problem Formulation}

During the price manipulation period, a significant price change is the expected effect of the spoofing orders by the manipulators. The change is not realized incidentally by heuristic attempts of order submission but by careful designs based on the market microstructure theories, called market impact, which is defined as the impact of the trader's own actions on the market [19]. The quantitative research in [20] estimated that a quote that is even slightly higher (5 bps) than the bid price can induce a further 5.95 bps increase of the market best bid price. For normal traders, measuring and eliminating the market impact is crucial for trading models [19]. However, for the market manipulators, the market impact is what they use in the strategies for making economical profit.

The documented manipulation case of quote stuffing shown in Fig. 2(a), showed an actual market impact scenario. After placing a bona fide sell order at an expected price, a number of nonbona fide buy orders with quotes successively higher than the best bid price were submitted to make it appear that there was active buying interest on that equity. Somebody who was encouraged by the fictitious bid price changes responded to the bona fide sell order. Immediately after the sell order was nearly executed, the previous bid quoting orders were all cancelled, which steeply dropped the bid price to its initial level. To minimize the risk of the buy spoofing orders being unintentionally picked up by other investors, the manipulation process usually occurred within an extremely short time period. By the sequential quotes and quick cancellations, the nonbona fide orders in Fig. 2(a) made a sawtooth shaped 
market impact on the bid price. Thus, this price manipulation case can be illustrated as an instantaneous sawtooth pattern on the bid price time series. The sawtooth pattern, also discovered in [11] and [21], was identified as one of the highlighted patterns on bid/ask price time series during manipulation periods [13].

Similarly, the market impact triggered by another price manipulation type, ramping, can also be illustrated by special patterns on bid/ask price time series. Unlike the activities of quote stuffing, in ramping, one single spoofing order was usually placed inside the bid ask spread (higher than the current bid or lower than the current ask). By this activity, the manipulated bid/ask price can be moved a small percentage and reverted to its prior level in tiny time intervals. Small square wave fluctuations were then shown [Fig. 2(c)] as the pattern of ramping [22], which can also occasionally be a significant movement, as shown in Fig. 2(b). Although this stunning pulse rising is rare since most exchange markets such as Euronext NSC suspend continuous trading if prices change by more than $2 \%$ within the defined interval (e.g., 1 s) [20], it is highlighted as one of the typical manipulation cases in [21].

The sawtooth, square wave, and pulse (Fig. 2) represent the intrinsic patterns corresponding to the primary manipulation tactics. A mixture of such patterns have been found in real manipulation cases [11]. Consequently, the problem of detecting price manipulation activities can be transformed to the problem of detecting unusual/anomalous patterns in the bid/ask price time series. This transformation also corresponds with the stock-volatility rules released in April 2013 by SEC in the USA, which allows trading only within certain price bands to avoid unusually volatile trading behaviors [23]. Since the manipulation activities usually occur in tiny time intervals within one single trading day, the scope of our problem is to detect unusual/anomalous patterns in intraday bid/ask price time series. The time scale is discretely measured in terms of bid/ask price update event. Hence, the adjustment speed measured in physical time ultimately depends on the underlying frequency of order activities and differs across the market.

In the financial area, it is often believed that the nature of the bid/ask price follows the mean-reverting feature, the tendency to randomly oscillate away from and, over time, back toward an equilibrium price level determined by the long-term mean of the equity [24]. Studies that support the mean-reversion features can be traced back to 1930s in the empirical studies in [25] and then furthered in [26] and [27].

The price fluctuations triggered by the manipulation strategies are merely the unusual short-termed momentary oscillations with small amplitudes around the equilibrium level of the price [6.9 bps in Fig. 2(a) and 18.6 bps in Fig. 2(c)]. Those tiny oscillations are usually considered as the contamination of the financial data [28], [29]. For studies of financial time series, it is very important to reveal the true signal from the financial data series [29], which contains short, high-frequency noisy transients as well as long, low-frequency movement. For price manipulation detection, the high-frequency noisy parts are where the manipulation patterns hide. Accordingly, retrieving and directly analyzing those high-frequency components as well as the original price information might help develop an effective detection model.

\section{Characterizing Price Manipulation}

The detection system proposed in this paper is comprised of a feature extraction module and a detection model, where the model is trained based on the features extracted from the equity bid/ask price by the feature extraction module.

\section{A. Price Manipulation Features}

The three patterns that characterize typical price manipulations can be defined in two ways: remarkable pulse [Fig. 2(b)] or short-term small fluctuations [sawtooth and square in Fig. 2(a) and (c)]. The intuitive feature of the three patterns can be extracted as the rate of price (bid/ask) change, which is defined as the first order derivative of $P(t)$ with respect to $t$, $d P(t) / d t=\lim _{\Delta t \rightarrow 0} P(t+\Delta t)-P(t) / \Delta t$, where $\Delta t$ is the time interval between the changes of the ask/bid prices.

Empirical mode decomposition (EMD), continuous wavelet transform (CWT), and synchrosqueezed transform (SST) are commonly used for signal decomposition. EMD is limited by its rather low frequency resolution [30] and the obtainable frequency resolution of EMD depends on a critical frequency limit [31]. CWT can map any signal to a set of base functions obtained through dilation and translation of a mother wavelet, and it is able to achieve decomposition of the signals in different frequency bands and at different time points [32]. Unlike Fourier transform, which gives a representation of frequency content of a signal without time information, the time-frequency localization feature of CWT describes the frequency content locally in time by scale and shift parameters [32]. SST extends the CWT by reallocating the wavelet coefficients through further calculating the first derivative (instantaneous frequency) of the coefficients, which is named as synchrosqueezing [33]. SST has been recently applied as a bandpass filter for removing high frequency components from a signal in [34]. In our work, to retrieve and analyze the high-frequency components of the bid/ask prices, wavelet transform is applied as a feature extraction approach due to its wide and successful application in finance [29], [35].

The wavelet decomposition of a signal results in levels of approximation coefficients and detailed coefficients. The approximation coefficient vector reflects the low-frequency features and the detail coefficient vector reflects the highfrequency component of the signal. To retrieve the high frequency components and remove the low-frequency equilibrium component, the detail coefficient hard thresholding method, which is usually applied in financial signal de-noising [29], is applied inversely, so that the wavelet coefficients outside the thresholds are set to zeros

$$
T_{m, n}=\left\{\begin{array}{lll}
=T_{m, n} & \text { if } & \left|T_{m, n}\right| \leq \lambda \\
=0 & \text { if } & \left|T_{m, n}\right|>\lambda
\end{array}\right.
$$

where $T_{m, n}$ is the detail coefficient, $m, n$ are scale and shifting parameters of the wavelet function, respectively, and $\lambda$ is the fixed threshold. The wavelet and inverse thresholding procedure consist of three steps: 1) calculate the wavelet coefficient 


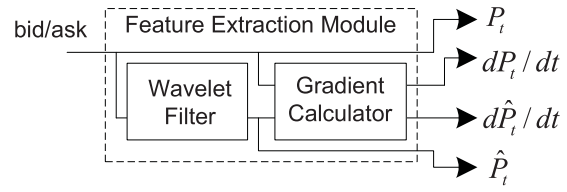

Fig. 3. Feature extraction module.

vectors $\left\{T_{m, n}, T_{m-1, n}, \ldots, T_{1, n}\right\}$ by discrete wavelet transform; 2) select a threshold $\lambda$ and filter the coefficient vectors by through the hard thresholding in (1); and 3) recompose the data using the filtered wavelet coefficients. In this paper, Symlets wavelet is chosen and applied for extracting the high frequency components (denoted by $\hat{P}_{t}$ ) of an equity price $P_{t}$. Since the short-term small fluctuation is only associated with high frequency components, we also refer to $\hat{P}_{t}$ as the shortterm small fluctuation.

\section{B. Features Extraction}

The first-order derivatives of the original price $P_{t}$ as well as the short-term oscillation $\hat{P}_{t}$ with respect to time are extracted as the features of the patterns of price manipulation. To capture this, the gradient of $P_{t}$ and $\hat{P}_{t}$ are all calculated. Among the methods for calculating gradients, finite difference is chosen and its central difference form is used since it is widely used in the finance area [36]: $d f(t) / d t \approx f(t+1 / 2 \Delta t)-f(t-1 / 2 \Delta t) / \Delta t$, where $f(t)$ represents $P_{t}$ and $\hat{P}_{t}$ and $\Delta t$ is the time interval between the changes of ask/bid prices. As discussed in Section III-A, three representative manipulation patterns are defined by significant pulse [Fig. 2(b)] and short-term small fluctuations, where the former is associated with original price change rate $d P_{t} / d t$ and the latter mainly corresponds to the high-frequency oscillations $d \hat{P}_{t} / d t$.

Financial data usually contain low-frequency trend component upon which a variety of different frequencies are superimposed [34]. From a financial perspective, $d P_{t} / d t$ and $d \hat{P}_{t} / d t$ represent the features of the original and the de-trended (low-frequency component removed) prices, which intrinsically correlated with some typical price manipulation activities as previously discussed. Those two patterns that emerged with price manipulation activities persist regardless of the price manipulation forms (Table I). Simultaneously, $P_{t}$ and $\hat{P}_{t}$ are correlated with the unusual pulses and large square patterns.

Accordingly, all of the four values, $P_{t}, \hat{P}_{t}, d P_{t} / d t$ and $d \hat{P}_{t} / d t$, should be included as features to cover all the possible manipulation circumstances (sawtooth, pulse, and square patterns). The structure of the feature extraction module comprises a wavelet filter and gradient calculator and is shown in Fig. 3.

An example of the output of the feature extraction module is shown in Fig. 4. The bid/ask price $P_{t}$ is filtered by the Symlets wavelet at level 8 . The smoothed equilibrium values [shown by a dotted line in Fig. 4(a)] are removed and the shortterm oscillation $\hat{P}_{t}$ is extracted [Fig. 4(b)]. The gradients of $P_{t}$ and $\hat{P}_{t}$ are both calculated and shown in Fig. 4(c) and (d), respectively.

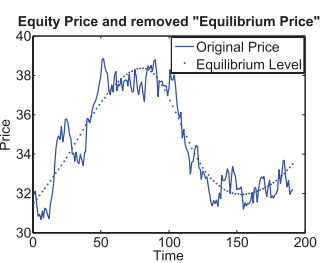

(a)

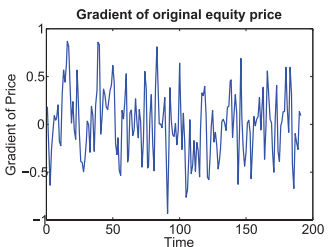

(c)

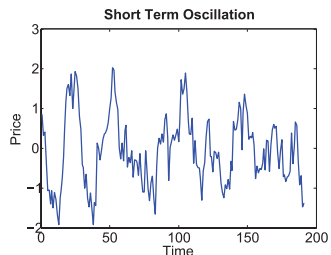

(b)

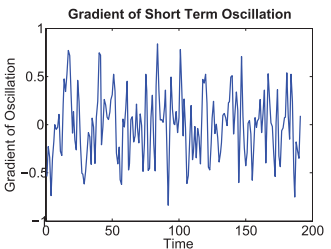

(d)
Fig. 4. Example of the equity price filtered by the wavelet. (a) Equity price and removed equilibrium price. (b) Short-term oscillation. (c) Gradient of original equity price. (d) Gradient of short-term oscillation.

\section{Detection Model}

Financial data are considered nonstationary in nature, meaning that the statistical properties (e.g., mean and variance) of the data change over time [37]. These changes are caused by various business and economic cycles in the longer term and demand-supply microstructures in the short term [38]. Of particular interest is the tendency of any unusual activities to exhibit a degree of non-stationarity of the extracted features. That is, the distribution of the extracted features changes over its duration. This may be interpreted as resulting from some of the irregular trading behaviors shown in Fig. 2. Hence, the detection model must be adopted to first capture the distributions of the extracted features and then detect the shifts in them.

\section{A. Gaussian Mixture Model and HMM}

A common solution for modeling the probability density function (pdf) of an observed variable is to approximate its unknown density with a Gaussian mixture model (GMM) [39]. A GMM is a weighted sum of $M$ component Gaussian densities as $p(\mathbf{x})=\sum_{i=1}^{M} w_{i} g\left(\mathbf{x} \mid \mu_{i}, \Sigma_{i}\right)$, where $\mathbf{x}$ is a $D$-dimension continuous-valued data vector, $w_{i}, i=1, \ldots, M$, are the mixture weights, and $g\left(\mathbf{x} \mid \mu_{i}, \Sigma_{i}\right)$, $i=1, \ldots, M$, are the component Gaussian densities. Each component density is a $D$-variate Gaussian function. Traditional GMM runs expectation maximization based on heuristic trials for the number of Gaussian components. To overcome this, a Dirichlet process GMM (DPGMM) [40], which provides a statistically principled manner for generating the number of the GMM components, is applied in our approach. DPGMM is the same as a regular GMM, except the component parameters drawn from a Dirichlet process as a prior probability [40] and the Gibbs sampling from the posterior probabilities [40].

In the price manipulation detection problem, the pdfs of extracted features of an equity price are modeled with DPGMM. The observed changes of the features can be 
detected by testing, which mixture component dominates the given value. This gives a simplified state view of the features. The temporal changes of distribution are probabilistically related to the changes of the observed features and the observed features sequences instead of any single values decide the potential manipulation. Such cases can be modeled by a HMM containing two sets of states, observable feature states and hidden mixture components states, which are assumed to depend only upon the previous states and modeled as a standard Markov process.

Usually, the application of a HMM in condition monitoring problems falls into two categories: signature modeling, where the detection model is learned from the activities to be recognized and the activities matching the model are reported as the detection results [41], [42], and anomaly detection, where a model of normality is learned from only the normal activities and the testcases against the model according to the predefined threshold are reported as anomalies [43], [44].

Due to the limited reports of market manipulation and regulatory rules prohibiting, the disclosure of data, which represents illegitimate trading behaviors, the availability of examples of market manipulation behaviors in capital market is far less than the availability of routine normal behavior. Consequently, price manipulation detection can be considered as an anomaly detection problem, which is the identification of new or unknown data patterns, to which a learning system has not been exposed during training. In this approach, normal bid/ask price patterns are modeled and the system will trigger an alarm when the behavior of the market action does not match with normal patterns. However, three problems arise in this approach when applied to price manipulation detection.

1) HMM is usually used to model 1-D sequential data rather than multiple features.

2) Anomaly detection using HMM usually lacks the ability of recognizing the anomaly type and the probability density of being that type.

3) The pdfs of the equity price evolve due to the nonstationary feature of financial time series.

To address these challenges, an improved adaptive HMM with anomaly states is developed and is presented in the following two sections.

\section{B. HMM With Anomaly States}

For a bid/ask price of an equity with $T$ points, four features are extracted by the module shown in Fig. 3. Letting $\boldsymbol{F}_{t},(t=$ $1, \ldots, T)$ be the feature vector at the $t$ th point in time, the preprocessed four features at time $t, \boldsymbol{F}_{t}=\left[F_{t}^{o}, F_{t}^{o g}, F_{t}^{s}, F_{t}^{s g}\right]$, are described as follows:

1) original price: $F_{t}^{o}$;

2) gradient of the of original price: $F_{t}^{o g}$;

3) short-term fluctuation: $F_{t}^{s}$;

4) gradient of the short-term fluctuation: $F_{t}^{s g}$.

The pdfs of the four features are learned separately by the GMM. Since the pdf of each feature might have multiple Gaussian components, the changes of the observation distribution can be detected by testing, which mixture component in the pdf was most likely to have generated a given value. Those

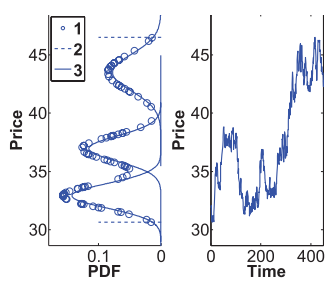

(a)

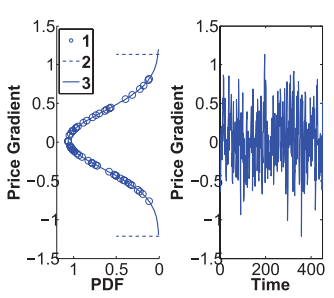

(c)

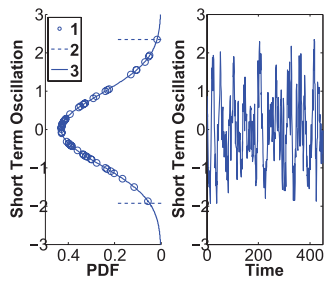

(b)

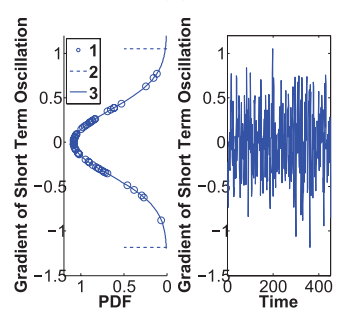

(d)
Fig. 5. Example of the features learned by GMM. The pdf of original equity price, the short-term oscillation, the gradient of original equity price and the gradient of oscillation are plotted (Legend: 1: pdf. 2: Components of pdf. 3: thresholds). (a) Equity price (right) and pdf (left). (b) Short-term oscillation (right) and its pdf (left). (c) Gradient of original equity price (right) and its pdf (left). (d) Gradient of short-term oscillation (right) and its pdf (left).

mixture components are then designated as hidden states of the observed equity price. This gives a simplified state view of time series data.

For the learned pdfs, anomaly thresholds separating the normal and anomaly are set according to the minimum acceptable data-likelihood value, which is adopted from the industry reference detection algorithm from Smart Group [17]: the $99 \%$ cumulative distribution cutoff. That means, the data are accepted as normal for which $P\left(\boldsymbol{F}_{t}\right) \geq 99 \%$. This is to identify the highest and lowest $0.5 \%$ frequent values for each feature. This heuristic method is also applied in a one-class support vector machine (OCSVM) [45], where the detection boundary is usually set to include most (e.g., 99\%), but not all, training data to avoid high miss detection rate. By doing this, the $1 \%$ extreme values are not simply assumed to be abnormalities but generate the dummy anomaly states for the HMM.

Fig. 5 shows an example of the pdfs of four features of the time series data. The pdfs of the features are learned by the GMM. Two thresholds (shown by dotted lines) are set to $0.5 \%$ and $99.5 \%$ cumulative probability of each pdf, separating the normal and anomaly regions. Fig. 5(a) shows a pdf of an equity price (illustrated by the small circles) with three Gaussian components (illustrated by the solid line). The thresholds (illustrated by the dotted lines) are set such that the $99 \%$ cumulative distribution of the equity prices are accepted as being normal leaving the maximal and minimal $0.5 \%$ as being anomalies. By this, the pdf of the original equity price can be partitioned into four parts: three parts corresponding to three Gaussian components in the normal region and one corresponding to the regions with anomalies values (both maximal and minimal). The four parts can then be designated as four hidden states respectively for the equity price feature, as shown in Fig. 6. Defining those hidden states 


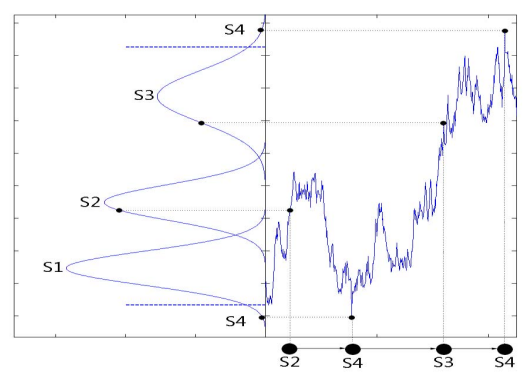

Fig. 6. HMM with anomaly states generated from a sequence of observation of variable. S1, S2, and S3 are normal states and S4 is the abnormal state.

as $\left\{f_{j_{o}}^{o}, j_{o}=1, \ldots, 4\right\}$, we have that the extracted feature $F_{t}^{o} \in\left\{f_{j_{o}}^{o}, j_{o}=1, \ldots, 4\right\}$.

Similarly, two hidden states (one anomaly and one normal) are generated for another three one-component pdfs [shown by Fig. $5(\mathrm{~b})-(\mathrm{d})]$. We can also define $\left\{f_{j_{o g}}^{o g}, j_{o g}=1,2\right\}$, $\left\{f_{j_{s}}^{s}, j_{s}=1,2\right\}$ and $\left\{f_{j_{s g}}^{s g}, j_{s g}=1,2\right\}$ as the hidden states of price gradient, short-term oscillation and oscillation gradients, respectively. We also have $F_{t}^{o g} \in\left\{f_{j_{o g}}^{o g}\right\}, F_{t}^{s} \in\left\{f_{j_{s}}^{s}\right\}$ and $F_{t}^{s g} \in\left\{f_{j_{s g}}^{s g}\right\}$, where $j_{o g}, j_{s}, j_{s g}=\{1,2\}$.

The hidden states of all four features are then combined as the quantized hidden states of the HMMAS model so that $4 \times 2 \times 2 \times 2=32$ hidden states are generated.

The quantized 32-state view of the observed bid/ask price can be represented as $\mathbf{S}=\left\{S_{i}\right\}$; where $S_{i}=$ $\left[f_{j_{o}}^{o}, f_{j_{o g}}^{o g}, f_{j_{s}}^{s}, f_{j_{s g}}^{s g}\right] ; \mathbf{S}$ is a collection of the feature states $S_{i}$, $i \in\{1, \ldots, 32\}, j_{o} \in\{1, \ldots, 4\}$ and $j_{o g}, j_{s}, j_{s g} \in\{1,2\}$. Each hidden state contains a unique combination of the feature status. The state having all features within the normal region is considered as the completely normal hidden state and others having at least one feature within the anomaly region are considered anomaly hidden states. Thus, only three among 32 states are normal while 29 are anomaly.

According to the official definitions of the price manipulation in market abuse directive [9] and the discussion in Section II, the typical price manipulation types are associated with the combinations of different features status. The types, quote stuffing, momentum ignition, and gouging, which are featured by instantaneous sweeping of order books, are essentially associated with the significant changes of gradients of short-term oscillations and the original price $\left(f_{j_{s g} g}^{s g}\right.$ and $\left.f_{j_{o g}}^{o g}\right)$ rather than the large price fluctuation. Besides, the types, ramping, pump and dump, capping and pegging, and near close are primarily associated with significant changes of the original price or short-term fluctuations $\left(f_{j_{o}}^{o}\right.$ and $\left.f_{j_{s}}^{s}\right)$ but not necessarily related to the gradients.

Consequently, according to the features status, the 29 anomaly states can be combined into three manipulation states, namely: quote stuffing, ramping, and other anomalies. The states with only anomalous gradient features fall into the quote stuffing while the states with only the anomalous price features (original and the oscillation) are ramping. The manipulation state named other anomalies is associated with the ones in which all of the features are anomalous. By this merging, the 32 states are further simplified as a six-state view of

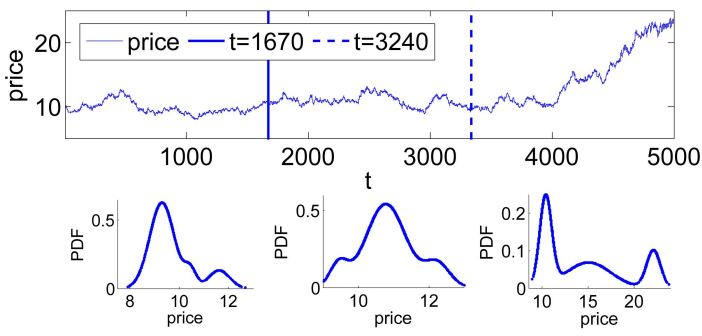

Fig. 7. Nonstationary changes shown by different pdfs.

the observed bid/ask price with three normal states and three anomaly states referring to primary manipulation types. Hence, the six hidden states provide a complete spectrum as well as an efficient way for representing the bid/ask price shifting triggered by the trading behaviors, where, for brevity, only the transfers in hidden states are shown rather than the observed temporal dynamics on each feature. In this way, the traditional HMM is extended to comprise anomaly states that cannot be otherwise achieved, hence the name HMMAS.

The basic property of HMMAS, inherited from the traditional HMM, is the probabilities yielded by the Viterbi algorithms, which determines from an observation sequence the most likely sequence of underlying hidden states that might have generated it. Together with the probability of an observed sequence, HMMAS provides a measure of specific price manipulation type identification according to the states and the features.

\section{Adaptive HMMAS}

Due to the nonstationary feature of financial time series, the mean and the variance of the pdfs of the equity price might vary over time. To deal with the nonstationarity of the time series of bid/ask price, the HMMAS is improved by an adaptive mechanism [and renamed the AHMMAS], where the model is trained using the data in previous specific time periods, defined as a sliding window with length $w$. During the course of detecting, the price manipulation activities, the window is slid forward to maintain the closest $w$ data points and AHMMAS is updated if significant discrepancies between the $w$ data points in the current sliding window and the prior training data are detected.

The deviation between the two data sequences is detected using the t-test. A simple example of the nonstationarity of equity price is shown in Fig. 7. Recall that a process is strongly stationary whenever its distribution is invariant under time shifts [46]. Part of the Apple stock bid price on 12 June 2012 $P_{t}, t=1, \ldots, 5000$ is shown in Fig. 7. If the sliding window length is set to $w=1670$, the variant pdf of the time series in three windows $P_{t}, t=1, \ldots, 1670, P_{t}, t=1671, \ldots, 3240$, and $P_{t}, t=3241, \ldots, 5000$ (shown as the three parts in Fig. 7) are detected as significant changes of the statistical features of the time series by $t$-test under the significance level $1 \%$. A $t$-test module is then designed as a postprocessing block of AHMMAS for detecting the statistical difference between the updated data sequence in the sliding window and the previous training data sequence and a popular significance level, $1 \%$, 


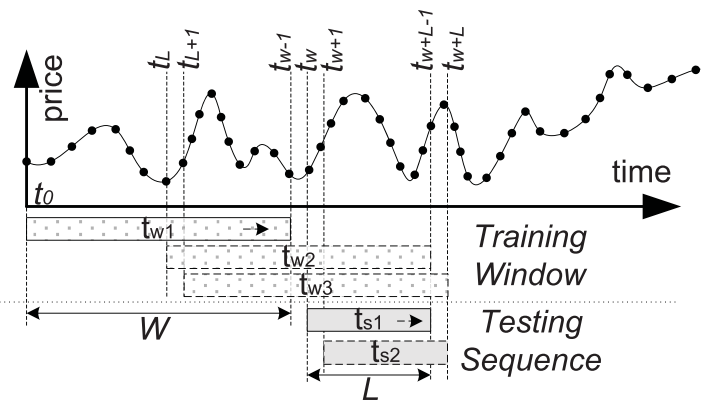

Fig. 8. Training and testing mechanism of price manipulation detection system.

is chosen for balancing the tradeoff between the computation load and the performance.

The adaptive mechanism of AHMMAS is, as shown in Fig. 8. The model is initially trained by $w$ data points in the training window $\left(\boldsymbol{F}_{t}, t \in\left[t_{s 1}, t_{e 1}\right]\right.$, where $t_{s 1}$ and $t_{e 1}$ are the starting and ending time points, respectively). A new incoming data point is then detected by the model. If detected as normal, it will be updated into the update window $\left(\boldsymbol{F}_{\boldsymbol{t}}, t \in\left[t_{s 2}, t_{e 2}\right]\right)$ for testing the statistical difference. As shown in Fig. 8, the data in the sliding window are tested every time the sliding window is updated, but the model might be retrained after working time period $t$. Since the scope of this paper is to detect the intraday price manipulation activities, the training window is set to one day.

\section{Detection Algorithm}

The algorithm for detecting the price manipulation activities by AHMMAS can then be summarized as per steps described in Algorithm 1.

Fig. 8 shows the initial states of the training window and the testing sequence and the sliding mechanism of each during the price manipulation detection process. In Fig. 8, the AHMMAS model was trained with the data in $t w_{1}=P_{t_{i}}^{E}, t_{i} \in[0, w-1]$. The first testing sequence was constructed as $t s_{1}=P_{t_{i}}^{E}, t_{i} \in$ $\left[t_{w}, t_{w+L-1}\right]$. If the detection indicate normal behaviors, the adaptive mechanism then checks for the significant changes between the data in the updated training window $t w_{2}=$ $P_{t_{i}}^{E}, t_{i} \in\left[t_{L}, t_{w+L-1}\right]$ and the original training window $t w_{1}$. If no retraining is required, the training window will slide forward as $t_{w}$ and a new testing sequence will be constructed as $t s_{2}$ to include the new data point at $t_{w+L}$ and exclude the point at $t_{w}$. Similarly, if no anomaly is detected, the data in the updated training window $t w_{3}=P_{t_{i}}^{E}, t_{i} \in\left[t_{L+1}, t_{w+L}\right]$ and in the original training window $t w_{1}$ are detected for adaptation. This detection and updating process continues for all incoming data streams.

\section{EXPERIMENTS AND EVALUATION}

Evaluating a detection model usually relies on the labeled benchmarks of both normal and anomaly cases. Since the absence of the real market anomaly cases, to evaluate the proposed detection model, it is acceptable to the financial industry business that all the characteristic patterns of reported manipulation examples are studied and then reproduced in
Algorithm 1 Price Manipulation Detection

Step 1: For the specific equity $E$, select a sliding window length $w$; Construct the bidlask price time series $P_{t}^{E}$ from $t_{0}$ to the current time point $t_{w-1}: P_{t_{i}}^{E}, t_{i} \in\left[t_{0}, \ldots, t_{w-1}\right]$;

Step 2: Calculate the four feature sequences of $P_{t_{i}}^{E}$ using wavelet and gradient approaches described above; obtain the features vector $\boldsymbol{F}_{t_{i}}=\left[F_{t_{i}}^{o}, F_{t_{i}}^{o g}, F_{t_{i}}^{s}, F_{t_{i}}^{s g}\right]$.

Step 3: Construct the AHMMAS detection model:

1) Calculate the pdf separately for the features, $F_{t_{i}}^{o}, F_{t_{i}}^{o g}, F_{t_{i}}^{s}, F_{t_{i}}^{s g}$, using the GMM and set the corresponding anomaly thresholds;

2) Construct the substates for each attributes and then construct the hidden states by combining four attributes according to discussion in Section IV-B and the illustration shown in Fig.6.

Step 4: Train the AHMMAS model using the observed features and the constructed hidden states.

Step 5: If the testing sequence is not constructed: select a detection sequence length $L$; the first testing sequence is then constructed as $P_{t_{i}}^{E}, t_{i} \in\left[t_{w}, t_{w+L-1}\right]$.

1) calculate the four features of the sequence using the wavelet and gradient blocks,

2) feed the features into AHMMAS model for calculating the probability of being specific states.

Step 6: If the state is identified as any anomaly states, the alert is triggered with the identified possible manipulation type and the probability of being the type.

Step 7: If the state is among the normal states, the bidlask price in sliding window $t_{L}$ to $t_{w+L-1}$ are fed to the adaptive mechanism for model updating check. If model updating is needed, the algorithm flow goes to Step 1 .

Step 8: If the model updating is not needed, the testing sequence is afterward updated as $P_{t_{i}}^{E}, t_{i} \in\left[t_{w+1}, t_{w+L}\right]$ and the algorithm flow goes to Step 5.

other financial data context to synthesize exploratory manipulation cases [47]. Synthetic exploratory financial data are also accepted in academia for evaluating the proposed model when real market data are hard to collect [48]-[50]. Thus, in this approach, the manipulation cases are synthetically generated and injected in any financial time series data while maintaining the normal statistical features such as the mean, variance, and volatility.

\section{A. Experimental Setup}

The experimental data used in this paper involves two categories. The data examples in the first category involve real market data of seven representative stocks: Google, Microsoft, Intel, and Apple from NASDAQ (obtained from the LOBSTER project [51]) and ARM, BARCLAYS, and Vodafone from London Stock Exchange. The selection of these data sets is due to their relatively high trading volumes and price volatility, the factors that might increase the likelihood of manipulation across the exchanges [18], [52]. This data set covers tick data over five trading days (June 11-15, 2012), and consists of 


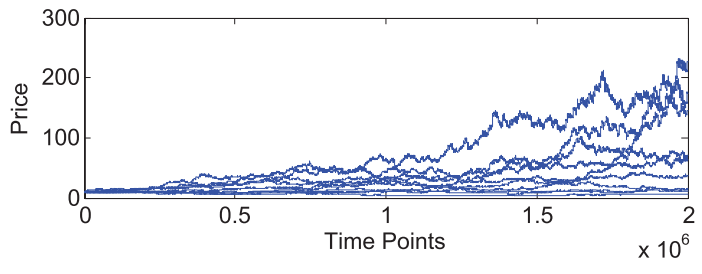

Fig. 9. 10 simulated prices paths.

more than 2000000 data points in total for each stock. The data sets have been examined by our financial partners to guarantee that no data are related to any reported manipulation cases by the regulators. Additionally, a statistical test widely used in trading firms [11] is performed on those data sets targeting intraday volatility anomalies. The testing is defined as: an anomalous pattern starting at time $t$ if $\sigma_{t-s, t} \leq 2 \bar{\sigma}$ and $\sigma_{t, t+s}>2 \bar{\sigma}$, where $s$ is defined as $1 \mathrm{~h}, \sigma_{t_{1}, t_{2}}$ is the volatility over the period $\left(t_{1}, t_{2}\right)$, and $\bar{\sigma}$ is the average intraday volatility across the whole data set. According to the manual examination and testing, no manipulation as well as volatility spikes are involved in those data sets.

The data examples in the second category are simulated stock prices generated using the stochastic differential equation (SDE): $d S_{t}=A\left(L-S_{t}\right) d t+$ stochastic term, where $A$ is the rate of the mean reversion, $L$ is the value around which the process $S_{t}$ tends to oscillate, and the stochastic term is a Brownian motion process. The end result is that the stock price tends to be a modulated nonstationary process of periodic waves and stochastic oscillations [53]. It should be noted that the price is not assumed to be $100 \%$ following the SDE but is merely to test the validity and robustness of the detection model under any nonstationary randomness of equity processes. In this category, there are 10 simulated prices paths, each containing 2000000 time points, as shown in Fig. 9.

Three typical manipulation patterns, shown in Fig. 2, are reproduced within each equity following the original characteristics: 6.9-bps sawtooth patterns in $819 \mathrm{~ms}, 18.6$-bps square wave in $0.1 \mathrm{~s}$ and 800 -bps pulse in $1 \mathrm{~s}$. Those anomalies are then injected into the corresponding time series making the test data a mixture of both normal and anomaly patterns. The trained models are deployed on the mixed test data to detect anomaly patterns. This is practical and acceptable to business people [54], especially when it is very costly to obtain real manipulation cases.

Since the length of the sliding window for the adaptive mechanism is set to be one day, for the real market data in the first testcase category, the data on June 11, 2012, the first day among the five, are chosen as the training data set, which contains around 400000 points. The fivefold cross-validation is used on the 400000 data points for training the detection model. The data from June 12-15, 2012 are set as the testing data set. Similarly, for the simulated stock price in the second category, the data are equally partitioned into five sets, each of which contains exactly 400000 points. The training data set consists of the first set and the testing data set consists of the other four sets. The five-folder cross-validation is used on the training data set for constructing the detection model. Since the manipulation activities usually occur in an extremely short time period, the testing sequence length $L$ (Fig. 8) is set to 1 min, which is sufficient for one or two manipulation patterns.

To ensure comprehensive assessment of the approach, multiple testing data sets are built on both real market data and simulated data. For real stock prices, 300 synthesized patterns are injected to each data set with each type having 100 examples (one pattern in Fig. 2 is considered as one anomaly example.). For the simulated stock prices, five groups of testcases $n=1, \ldots, 5$ are generated and injected to each data set, where group $n=1$ contains 10 (examples/type) $\times 3$ (types) $=$ 30 total numbers of injected anomaly examples; group $n=2$ has $20 \times 3=60$ numbers and group $n=3,4,5$ has $40 \times 3=$ $120,80 \times 3=240$, and $100 \times 3=300$ total numbers of injected anomaly examples, respectively.

As discussed in Section II-B, some generic computational models were used for manipulation detection such as SVM, neural network and logistic regression in [14] and [15] as well as the rule-based algorithms in [16] and [17]. Since the rulebased algorithms were specific for the special manipulation scenarios discussed in [16] and [17], they are not suitable for general manipulation detection problems. According to those related work, we choose three popular computational models as the benchmarks of our model: OCSVM [45], $k$ nearest neighbor (kNN) [39], and GMM [39]. Those benchmark models are applied on the same feature vector $F_{t}=$ $\left[F_{t}^{o}, F_{t}^{o g}, F_{t}^{s}, F_{t}^{s g}\right]$. In our experiments, LIBSVM [55] and DDTool [56], are used as the implementation of the benchmark models. All the model parameters (i.e., kernel width for OCSVM, $k$ value for kNN, and the number of components for GMM) are fine-tuned through five-folder cross-validation on each different data set for stable and optimized testing results.

The performance evaluation of the proposed model is based on two types of metrics, the receiver operating characteristic (ROC) and F-measure. Both of them are based on the confusion matrix, where false positive (FP), is defined as manipulation cases detected as normal and false negative (FN) is defined as normal cases detected as manipulation, true positive (TP) is defined as normal cases detected as normal, and true negative (TN) is defined as manipulation cases detected as manipulation. The ROC curve is a widely used metric for evaluating and comparing binary classifiers [57]. The ROC curve plots the true positive rate [(TPR) $(\mathrm{TP} / \mathrm{TP}+\mathrm{FN})]$ against the false positive rate $[(\mathrm{FPR})(\mathrm{FP} / \mathrm{FP}+\mathrm{TN})]$ while the discrimination threshold of the binary classifier is varied. To assess the overall performance of a binary classifier, one can measure the area under the Area Under ROC curve (AUC). The maximum value of AUC is 1 and therefore larger AUC values indicate generally better classification performance. The ROC curve and AUC are used as the performance measure on seven real stock data sets. The F-measure integrates both precision and sensitivity into one single metric and widely used in anomaly/novelty detection area [58]. The F-measure is used for evaluating the testing performance on simulated data sets.

\section{B. Experimental Results}

The ROC curves of four models on seven real-stock data sets with $3 \times 100=300$ numbers of injected novelties in 

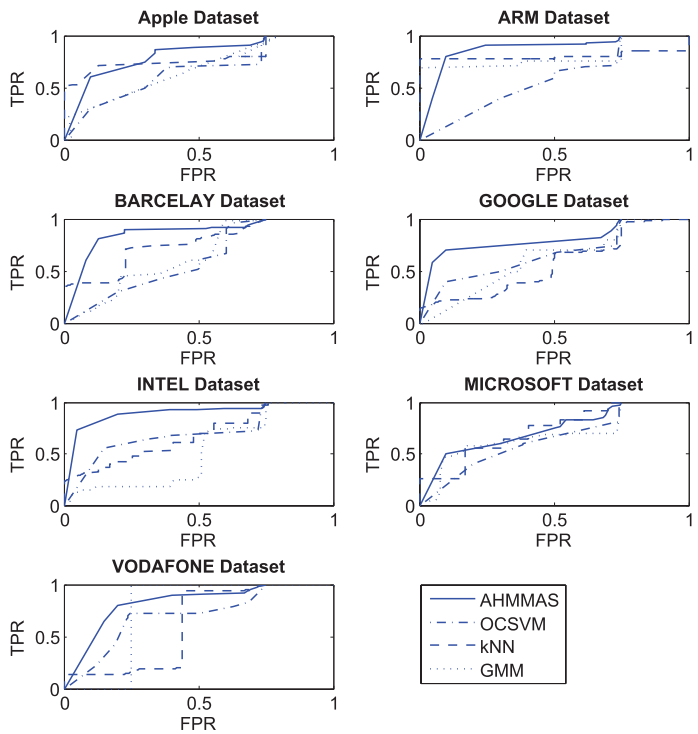

Fig. 10. ROC of four models on seven stock data sets (TPR and FPR).

TABLE II

aUC of Four Detection Models on Seven Real Stock Data Sets

\begin{tabular}{l|cccc}
\hline \hline AUC & AHMMAS & OCSVM & kNN & GMM \\
\hline AAPL & $\mathbf{0 . 8 1 4 2}$ & 0.6603 & 0.7926 & 0.6695 \\
ARM & $\mathbf{0 . 8 2 7 0}$ & 0.5830 & 0.7982 & 0.7918 \\
BARC & $\mathbf{0 . 8 7 1 0}$ & 0.6125 & 0.7627 & 0.6466 \\
GOOG & $\mathbf{0 . 8 0 2 5}$ & 0.6593 & 0.5612 & 0.6163 \\
INTC & $\mathbf{0 . 8 9 7 1}$ & 0.6970 & 0.6280 & 0.5200 \\
MSFT & $\mathbf{0 . 7 3 3 6}$ & 0.6419 & 0.6250 & 0.6802 \\
VOD & $\mathbf{0 . 8 7 7 5}$ & 0.7044 & 0.7278 & 0.7495 \\
\hline \hline
\end{tabular}

each data set are shown in Fig. 10. To compute such a curve, the discrimination thresholds are set from 0.1 to 0.9 with increment, 0.01, for each model. The calculated AUC values for four models are summarized in Table II.

The AHMMAS model achieved the highest AUC on all of the seven real stock price data sets and it outperforms the benchmark models. The best AUC value for AHMMAS model appears on the Intel data set $(0.8971)$, which is $22 \%$, $29 \%$, and $42 \%$ higher than OCSVM (0.6970), kNN (0.6280), and GMM (0.5200) models, respectively. The second best AUC value for AHMMAS model is on the Vodafone data set $(0.8775)$, which is around $15 \%-20 \%$ better than other three models. The lowest AUC value for AHMMAS model is on the Microsoft data set (0.7336) and is still 12\%, 14\%, and $7 \%$ higher than OCSVM (0.6419), kNN (0.6250), and GMM (0.6802) models, respectively. The performance difference between the AHMMAS model and other three models in terms of the AUC values are calculated on seven stock data sets as ( $\mathrm{AUC}_{\text {Aнmmas }}-\mathrm{AUC}_{\text {others }}$ )/AUC $\mathrm{AHMmas}_{\text {ah }}$ and are shown in Fig. 11, where the legends, OCSVM, kNN, and GMM, represent the performance differences between AHMMAS and OCSVM, $\mathrm{kNN}$, and GMM models, respectively.

The lowest value of the difference is between the AHMMAS and kNN models on Apple data set, where the AHMMAS model $(0.8142)$ performed $2.6 \%$ better than the $\mathrm{kNN}$ model (0.7926). The minimum value indicates the roughly identical performance of AHMMAS and kNN models on Apple

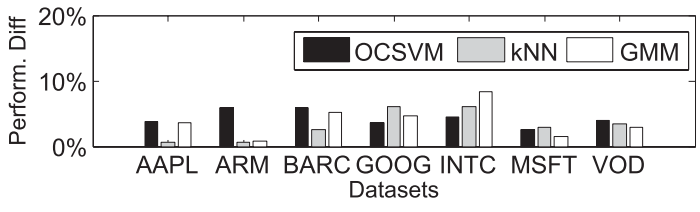

Fig. 11. Performance difference between AHMMAS and other models on seven data sets.
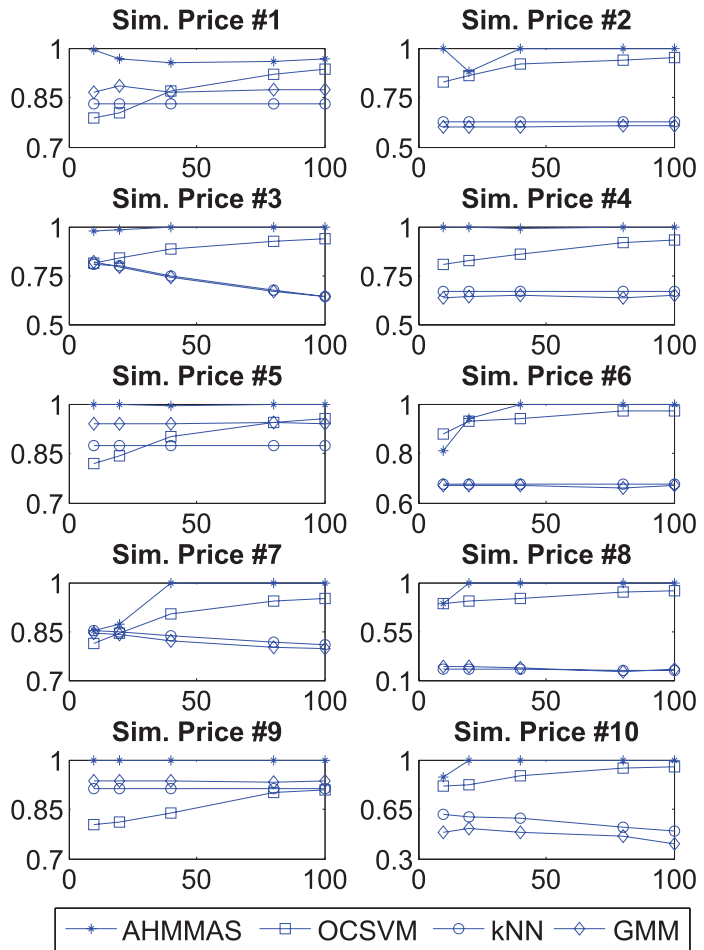

Fig. 12. F-measure of four models on simulated prices ( $x$-axis: number of anomalies. $y$-axis: F-measure).

data set. The largest value of the difference is between the AHMMAS and GMM models on Intel data set, where AHMMAS model (0.8971) performed $42 \%$ better than GMM model (0.5200). This is where AHMMAS performs the best among all the testcases. From Fig. 11, it is also obvious that the performance difference between AHMMAS and OCSVM models is relatively stable in range from $12 \%$ to $30 \%$ over different data sets while the other two performance differences are both volatile in range from $2 \%$ to $46 \%$.

The performance comparison shows that the AHMMAS model retains its advantage over all other models on seven real market data sets. It also indicates the performance stabilities of the three benchmark models, among which, OCSVM model performed relatively steady while the $\mathrm{kNN}$ and GMM model performed volatile across the different data sets.

The performance of the four models on 10 simulated prices data sets is evaluated based on F-measure metrics as shown in Fig. 12 where the horizontal axis represents the number of injected anomaly examples and the vertical axis represents the value of the F-measure. As discussed before, the models are tested on 10 simulated data sets, each of which contains five groups of injected anomaly examples. Thus, 50 different experiments are carried out as a robust evaluation plan for each model. 
From the results in Fig. 12, the AHMMAS model achieved the highest F-measure among the four models on most of the 10 data sets. In the case of simulated price \#6 with 10 injected anomaly examples, the initial F-measure for the AHMMAS model (0.8081) is lower than that of OCSVM (0.8791). However, the performance of AHMMAS increased on this testcase as the number of anomaly examples increased. Another case is on simulated price \#7 with 10 injected anomaly examples, the F-measure for AHMMAS model (0.8535) is very close to $\mathrm{kNN}$ model (0.8506) but increased on the testcases with 20 injected anomaly examples.

\section{Discussion}

The experiments on real and simulated price data sets have shown that the AHMMAS model outperforms the other three benchmark models: OCSVM, kNN, and GMM. The robustness can be explained by the inherited nature from HMM on time series data modeling, where the algorithm does not simplemindedly accept the most likely state for a given time instant, but takes a decision based on the whole sequence. Thus, if there are some particularly normal events midway through a sequence of anomalies, this will not matter provided the whole context of what is seen is reasonable. This is particularly valuable in price manipulation detection where normal and manipulation trading behaviors may be interleaved, but the overall sense of the events may be detectable. However, other detection models are trained for building tightly enclosed boundaries, which consider the testing examples as a set of separate points.

Another reason for the robust test results is the adaptive mechanism. Due to the nonstationary feature, the distribution of financial time series data $x_{t_{1}}, \ldots, x_{t_{l}}$ may deviate from $x_{t_{1+\tau}}, \ldots, x_{t_{l+\tau}}$. Detecting the statistically deviated data by a constant model increases the risk of obtaining incorrect detections. The mechanism of capturing the significant difference and updating the detection model provides a reasonable compensation for the nonstationarity.

The AHMMAS model performs distinctly on different data sets. In Table II, most of the AUC values for AHMMAS model go beyond 0.8 , which is considered as a threshold of good performance for a classifier [57]. However, the result for Microsoft data set is lower than others (0.7336) and is even $8.5 \%$ lower than the second lowest AUC (0.8025) on Google data set. Further investigation showed that the small AUC on Microsoft data set is due to the relatively high FN numbers (normal cases detected as anomaly) decreasing the TPR on each discrimination threshold. After examining the FN misclassification cases and comparing the training and testing data sets, it was discovered that the normal patterns in the testing data set that failed to be detected as normal were in fact identical to the anomaly patterns shown in Fig. 2 and were never seen in the training data set. In the example of the normal Microsoft testing data set (no anomaly pattern injected) shown in Fig. 13, the bid price from 8:00:00 to 8:01:00 A.M. on June 12,2012 . Oscillated unusually and showed mixed patterns of sawtooth and square wave, which are very easily identified as the anomalies by the detection model.

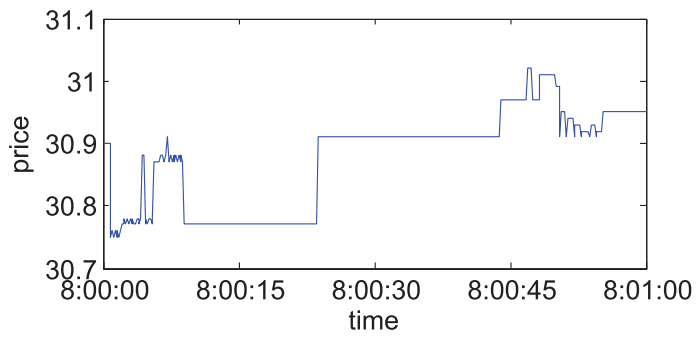

Fig. 13. Microsoft stock bid price from 8:00:00 to 8:01:40 A.M. on June 12, 2012. Normal data showed anomaly patterns.

Consultation with financial experts from industry suggested that although rarely, such normal but wild oscillation sometimes happen especially when experiencing high frequency trading (HFT). Although HFT is out of the scope of this paper due to its hyper-fast trading speed (e.g., 1000 updates in $1 \mathrm{~ms}$ ), the wild oscillations in Fig. 13 show analogous patterns as the manipulation algorithm triggered by HFT. The oscillation also conforms with the recent report from Nanex [59] that stock of Paychex showed the wild sawtooth oscillation when being hit by a HFT algorithm. The wild pattern is identical to the manipulation case documented in 2012 [Fig. 2(a)] although not reported by regulators yet. Therefore, although the FN cases (normal cases misclassified as anomaly) seem to decrease the detection performance, most of them referred to suspicious trading activities which are identical to the reported manipulation cases.

From the experimental results and the analysis of the misclassification, it can be concluded that the AHMMAS model is better suited for the price manipulation detection problem based on a bidlask price time series and its performance is consistently superior to OCSVM, kNN, and GMM models under most of the randomness of the nonstationarity of the underlying equity price.

Based on the consultations with financial experts, real-time surveillance in practice is required by the trading floor to recognize any unusual patterns, which are used as a reference to adapt their trading behaviors accordingly. Therefore, they do not conclusively determine the intention behind every anomaly or the contextual relation between those patterns but mitigate any possible negative impacts on their portfolios from the obvious problems in the market (e.g., the flash crash). Instead, the proposed method determines the manipulation by considering the anomalies as well as their contextual relations (through the Markov chain). The detection increases the accuracy while sacrificing the efficiency for real-time detection, which essentially may not be the first priority for regulators. Therefore, AHMMAS best suits the overnight detection in real world considering the computational complexity of the proposed method.

\section{CONCLUSION}

This paper presented a computational intelligence approach for price manipulation detection. Typical price manipulation cases were studied, where special patterns of the bid and ask price during a manipulation period were analyzed for extracting reliable features. Based on the extracted features, an AHMMAS was proposed for detecting the anomalies 
in the bid and ask prices. The AHMMAS considers the anomaly states according to the thresholds of four extracted features set by the pdfs of the features. A system has been developed and intensively tested on both real-life stock prices and simulated prices. The comparison of proposed approach with other benchmark models, OCSVM, kNN, and GMM, has shown that the AHMMAS performs better in terms of the area under ROC curve and the F-measure, respectively. Finally, the experimental results were analyzed and misclassification cases were discussed and further explained with examples.

In the AHMMAS model, the pdfs of the four extracted features are learned individually using DPGMM. However, the use of a joint-pdf may offer a more accurate approach for future studies since there is no empirical study showing the independence relationship of the four features and this will be the focus of our future work. Furthermore, modeling the bidlask time series with the corresponding order information may provide a mechanism for decreasing the FNR mentioned before. SST may also provide an enhanced signal decomposition technique by extending the traditional wavelet. Meanwhile, the exploration of coupled behaviors of the bid and ask prices of one stock might help to enhance the detection results of potential price manipulation strategies that target both bid and ask price for maximizing profits.

Additionally, the adaptive mechanism compensated the nonstationary features of the financial time series. However, the retraining processes increased the computational complexity. The rapidly growing trading frequency increased the nonstationarity of the time series and at the same time decreased the tolerance of latency for model retraining. However, an increased nonstationarity requires more retraining. To solve this contradictory problem, additional to the adaptive mechanism, one strand of the future works might be building a method that removes or partially removes the nonstationarity while maintaining the necessary statistical features.

\section{ACKNOWLEDGMENT}

The authors would like to thank Dr. P. McParland and Dr. B. Bland, Fidessa Group plc, for their support.

\section{REFERENCES}

[1] F. Allen and D. Gale, "Stock price manipulation," Rev. Financial Studies, vol. 5, no. 3, pp. 503-529, 1992.

[2] F. Allen, L. Litov, and J. Mei, "Large investors, price manipulation, and limits to arbitrage: An anatomy of market corners," Rev. Finance, vol. 10, no. 4, pp. 645-693, 2006.

[3] Y. S. Abu-Mostafa, A. F. Atiya, M. Magdon-Ismail, and H. White, "Introduction to the special issue on neural networks in financial engineering," IEEE Trans. Neural Netw., vol. 12, no. 4, pp. 653-656, Jul. 2001.

[4] B.-L. Zhang, R. Coggins, M. Jabri, D. Dersch, and B. Flower, "Multiresolution forecasting for futures trading using wavelet decompositions," IEEE Trans. Neural Netw., vol. 12, no. 4, pp. 765-775, Jul. 2001.

[5] T. Van Gestel et al., "Financial time series prediction using least squares support vector machines within the evidence framework," IEEE Trans. Neural Netw., vol. 12, no. 4, pp. 809-821, Jul. 2001.

[6] C. Bergmeir, I. Triguero, D. Molina, J. Aznarte, and J. Benitez, "Time series modeling and forecasting using memetic algorithms for regimeswitching models," IEEE Trans. Neural Netw. Learn. Syst., vol. 23, no. 11, pp. 1841-1847, Nov. 2012.
[7] A. Miranian and M. Abdollahzade, "Developing a local least-squares support vector machines-based neuro-fuzzy model for nonlinear and chaotic time series prediction," IEEE Trans. Neural Netw. Learn. Syst., vol. 24, no. 2, pp. 207-218, Feb. 2013.

[8] K. Menyah and K. Paudyal, "The components of bid-ask spreads on the London stock exchange," J. Banking, Finance, vol. 24, no. 11, pp. 1767-1785, 2000.

[9] Market Abuse, Amended Proposal for a Directive, European Commission, Brussels, Belgium, 2012.

[10] D. Cumming, S. Johan, and D. Li, "Exchange trading rules and stock market liquidity," J. Financial Economics, vol. 99, no. 3, pp. 651-671, 2011.

[11] J. Tse, X. Lin, and D. Vincent, "High frequency trading-Measurement, detection and response," Credit Suisse, Zürich, Switzerland, Tech. Rep., Dec. 2012.

[12] FINRA, Washington, DC, USA. (2012). Finra Joins Exchanges and the Sec in Fining Hold Brothers More Than \$5.9 Million for Manipulative Trading, Anti-Money Laundering, and Other Violations [Online]. Available: http://www.finra.org/Newsroom/NewsReleases/2012/P178687

[13] Nanex, Denver, CO, USA. (2012). Whac-a-Mole is Manipulation [Online]. Available: http://www.nanex.net/aqck2/3598.html

[14] H. Öğüt, M. M. Doğanay, and R. Aktaş, "Detecting stock-price manipulation in an emerging market: The case of Turkey," Expert Syst. Appl., vol. 36, no. 9, pp. 11944-11949, 2009.

[15] D. Diaz, B. Theodoulidis, and P. Sampaio, "Analysis of stock market manipulations using knowledge discovery techniques applied to intraday trade prices," Expert Syst. Appl., vol. 38, no. 10, pp. 12757-12771, 2011.

[16] J. Mongkolnavin and S. Tirapat, "Marking the close analysis in Thai Bond Market surveillance using association rules," Expert Syst. Appl., vol. 36 , no. 4, pp. 8523-8527, 2009.

[17] M. J. Aitken, F. H. Harris, and S. Ji, "Trade-based manipulation and market efficiency: A cross-market comparison," in Proc. 22nd Australasian Finance Banking Conf., 2009.

[18] E. J. Lee, K. S. Eom, and K. S. Park, "Microstructure-based manipulation: Strategic behavior and performance of spoofing traders," J. Financial Markets, vol. 16, no. 2, pp. 227-252, 2013.

[19] A. Ferraris, "Equity market impact models: Mathematics at the interface between business and research," Stifterverband fur die Deutsche Wissenschaft, Berlin, Germany, Tech. Rep., Dec. 2008.

[20] N. Hautsch and R. Huang, "The market impact of a limit order," J. Econ. Dyn. Control, vol. 36, no. 4, pp. 501-522, 2012.

[21] D. Ian. (2012). Market Abuse and Surveillance [Online]. Available: http://www.bis.gov.uk/assets/foresight/docs/computer-trading/121076-eia17-market-abuse-and-surveillance

[22] J. Tse, X. Lin, and D. Vincent, "High frequency trading-The good, the band and the regulation," Credit Suisse, Zürich, Switzerland, Tech. Rep., Dec. 2012.

[23] SEC, Atlanta, GA, USA. (2013). Securities and Exchange Commission Release No. 34-69287 [Online]. Available: http://www.sec.gov/rules/sro/nms/2013/34-69287.pdf

[24] H. Zhang and Q. Zhang, "Trading a mean-reverting asset: Buy low and sell high," Automatica, vol. 44, no. 6, pp. 1511-1518, 2008.

[25] A. Cowles and H. E. Jones, "Some a posteriori probabilities in stock market action," Econ., J. Econ. Soc., vol. 5, no. 3, pp. 280-294, 1937.

[26] C. M. Hafner and H. Herwartz, "Option pricing under linear autoregressive dynamics, heteroskedasticity, and conditional leptokurtosis," J. Empirical Finance, vol. 8, no. 1, pp. 1-34, 2001.

[27] L. P. Bos, A. F. Ware, and B. S. Pavlov, "On a semi-spectral method for pricing an option on a mean-reverting asset," Quantit. Finance, vol. 2, no. 5, pp. 337-345, 2002.

[28] R. R. Bliss and N. Panigirtzoglou, "Testing the stability of implied probability density functions," J. Banking, Finance, vol. 26, nos. 2-3, pp. 381-422, 2002.

[29] E. Haven, X. Liu, and L. Shen, "De-noising option prices with the wavelet method," Eur. J. Oper. Res., vol. 222, no. 1, pp. 104-112, 2012.

[30] Z. Wu and N. E. Huang, "A study of the characteristics of white noise using the empirical mode decomposition method," Proc. Roy. Soc., vol. 460, no. 2046, pp. 1597-1611, 2004.

[31] M. Feldman, "Analytical basics of the EMD: Two harmonics decomposition," Mech. Syst. Signal Process., vol. 23, no. 7, pp. 2059-2071, 2009.

[32] I. Daubechies, Ten Lectures on Wavelets, vol. 61. Philadelphia, PA, USA: SIAM, 1992, 
[33] I. Daubechies, J. Lu, and H.-T. Wu, "Synchrosqueezed wavelet transforms: An empirical mode decomposition-like tool," Appl. Comput. Harmon. Anal., vol. 30, no. 2, pp. 243-261, 2011.

[34] A. Ahrabian, C. Took, and D. Mandic, "Algorithmic trading using phase synchronization," IEEE J. Sel. Topics Signal Process., vol. 6, no. 4, pp. 399-404, Aug. 2012.

[35] M. A. L. Caetano and T. Yoneyama, "A new indicator of imminent occurrence of drawdown in the stock market," Phys. A, Statist. Mech. Appl., vol. 388, no. 17, pp. 3563-3571, 2009.

[36] J. C. Hull, Options, Futures and Other Derivatives, 8th ed. Upper Saddle River, NJ, USA: Pearson Education, 2011.

[37] R. Ghazali, A. J. Hussain, N. M. Nawi, and B. Mohamad, "Non-stationary and stationary prediction of financial time series using dynamic ridge polynomial neural network," Neurocomputing, vol. 72 , no. 10, pp. 2359-2367, 2009.

[38] F. Allen and G. Gorton, "Stock price manipulation, market microstructure and asymmetric information," Nat. Bureau Econ. Res., Cambridge, MA, USA, Tech. Rep. 3862, 1991.

[39] C. M. Bishop, Pattern Recognition and Machine Learning. New York, NY, USA: Springer-Verlag, 2006.

[40] R. M. Neal, "Markov chain sampling methods for Dirichlet process mixture models," J. Comput. Graph. Statist., vol. 9, no. 2, pp. 249-265, 2000.

[41] P. Guo, Z. Miao, X.-P. Zhang, Y. Shen, and S. Wang, "Coupled observation decomposed hidden Markov model for multiperson activity recognition," IEEE Trans. Circuits Syst. Video Technol., vol. 22, no. 9, pp. 1306-1320, Sep. 2012.

[42] L. Wang, M. Mehrabi, and E. Kannatey-Asibu, "Hidden Markov modelbased tool wear monitoring in turning," J. Manuf. Sci. Eng., vol. 124, no. 3, pp. 651-658, 2002.

[43] D. Y. Yeung and Y. Ding, "Host-based intrusion detection using dynamic and static behavioral models," Pattern Recognit., vol. 36, no. 1, pp. 229-243, 2003.

[44] D. A. Clifton, L. Tarassenko, C. Sage, and S. Sundaram, "Condition monitoring of manufacturing processes," in Proc. Condition Monitor. 2008, pp. 273-279.

[45] B. Schölkopf, J. Platt, J. Shawe-Taylor, A. Smola, and R. Williamson, "Estimating the support of a high-dimensional distribution," Neural Comput., vol. 13, no. 7, pp. 1443-1471, 2001.

[46] G. Grimmett and D. Stirzaker, Probability and Random Processes. New York, NY, USA: Oxford Univ. Press, 2001.

[47] NANEX, Denver, CO, USA. (2013). Exploratory Trading-Top 8 HFT Take Liquidity 59\% of the Time [Online]. Available: http://www.nanex.net/aqck2/4136.html

[48] G. K. Palshikar and M. M. Apte, "Collusion set detection using graph clustering," Data Mining Knowl. Discovery, vol. 16, no. 2, pp. 135-164, Apr. 2008

[49] Y. Ou, L. Cao, C. Luo, and C. Zhang, "Domain-driven local exceptional pattern mining for detecting stock price manipulation," in Proc. 10th Pacific Rim Int. Conf. Artif. Intell., Trends Artif. Intell., 2008, pp. 849-858.

[50] M. Franke, B. Hoser, and J. Schröder, "On the analysis of irregular stock market trading behavior," in Data Analysis, Machine Learning and Applications. Berlin, Germany: Springer-Verlag, 2008, pp. 355-362.

[51] LOBSTER, Atlanta, GA, USA. (2012). Limit Order Book System [Online]. Available: http://www.lobster.wiwi.hu-berlin.de

[52] D. J. Cumming, F. Zhan, and M. J. Aitken, "High frequency trading and end-of-day manipulation," York Univ., Toronto, ON, Canada, Tech. Rep., Oct. 2013.

[53] B. Avanzi and B. Wong, "On a mean reverting dividend strategy with Brownian motion," Insurance, Math. Econ., vol. 51, no. 2, pp. 229-238, 2012.

[54] L. Cao, Y. Ou, and P. Yu, "Coupled behavior analysis with applications," IEEE Trans. Knowl. Data Eng., vol. 24, no. 8, pp. 1378-1392, Aug. 2012.

[55] C. C. Chang and C. J. Lin, "LIBSVM: A library for support vector machines," ACM Trans. Intell. Syst. Technol., vol. 2, no. 3, pp. 1-27, 2011.

[56] D. Tax. (2012, May). DDtools, the Data Description Toolbox for MATLAB [Online]. Available: http://prlab.tudelft.nl/david-tax/dd_tools. html

[57] T. Fawcett, "An introduction to ROC analysis," Pattern Recognit. Lett., vol. 27, no. 8, pp. 861-874, Jun. 2006.
[58] Y. Tang, Y.-Q. Zhang, N. V. Chawla, and S. Krasser, "SVMs modeling for highly imbalanced classification," IEEE Trans. Syst., Man, Cybern. B, Cybern., vol. 39, no. 1, pp. 281-288, Feb. 2009.

[59] Nanex, Denver, CO, USA. (2013). There is No Stock Market Regulator [Online]. Available: http://www.nanex.net/aqck2/4183.html

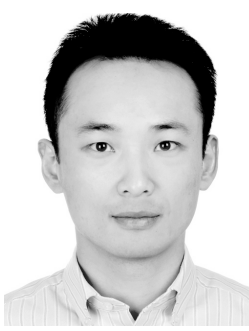

Yi Cao received the B.Eng. degree in navigation and control in aeronautics from the Beijing University of Aeronautics and Astronautics, Beijing, China, and the M.S. degree in computer science from Florida International University, Miami, FL, USA, in 2002 and 2005, respectively. He is currently pursuing the Ph.D. degree in computational finance with the University of Ulster, Londonderry, U.K.

He was an Integrated Circuit and Hardware Engineer at Vimicro, Beijing, and Conexant Beijing Design Centre, Beijing, respectively, from 2005 to 2008. From 2008 to 2011, he was a Senior System Engineer at ERICSSON, Beijing.

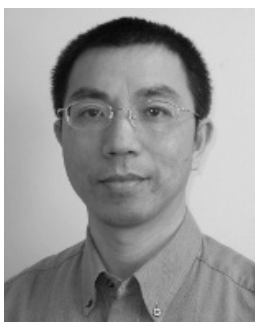

Yuhua Li (SM'11) received the Ph.D. degree in general engineering from the University of Leicester, Leicester, U.K.

He was with Manchester Metropolitan University, Manchester, U.K., and then the University of Manchester, Manchester, from 2000 to 2005, as a Senior Research Fellow and a Research Associate, respectively. Since 2005, he has been a Lecturer with the School of Computing and Intelligent Systems, University of Ulster, Londonderry, U.K. His current research interests include pattern recognition, machine learning, knowledge-based systems, signal processing, and condition monitoring and fault diagnosis.

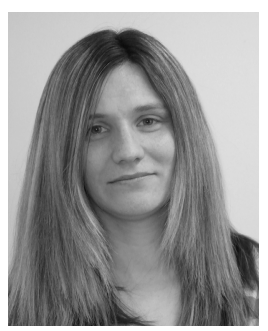

Sonya Coleman (M'11) received the B.Sc (Hons.) degree in mathematics, statistics, and computing, and the Ph.D. degree in mathematical image processing from the University of Ulster, Londonderry, U.K.

She is a Reader in the University of Ulster. She has authored and co-authored more than 80 research papers in image processing, robotics, and computational neuroscience. She has experience managing research grants (with respect to technical aspects and personnel) as both a principal and co-investigator. In addition, she is a co-investigator on the EU FP7 funded projects RUBICON and VISUALISE.

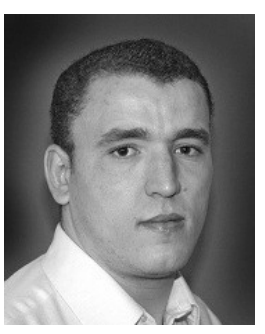

Ammar Belatreche received the Ph.D. degree in computer science from the University of Ulster, Londonderry, U.K.

He was a Research Assistant with the Intelligent Systems Engineering Laboratory and is currently a Lecturer with the School of Computing and Intelligent Systems, University of Ulster. His current research interests include bioinspired adaptive systems, machine learning, pattern recognition, and image processing and understanding.

Dr. Belatreche is a fellow of the Higher Education Academy, a member of IEEE Computational Intelligence Society (CIS), and the Northern Ireland representative of the IEEE CIS. He is an Associate Editor of Neurocomputing and has served as a PC Member and reviewer for several international conferences and journals.

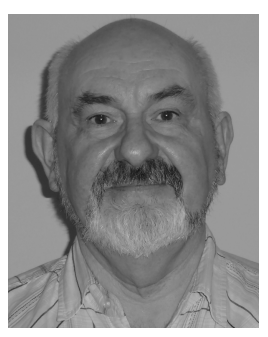

Thomas Martin McGinnity (SM'09) received the B.Sc. (Hons.) degree in physics and the Ph.D. degree from the University of Durham, Durham, U.K., in 1975 and 1979, respectivly.

He is currently a Dean of Science and Technology with Nottingham Trent University and was formerly a Professor of Intelligent Systems Engineering and Director of the Intelligent Systems Research Centre with the Faculty of Computing and Engineering, University of Ulster. He was a Director with the University of Ulster's technology transfer company, Innovation Ulster, and a spin out company Flex Language Services. He has authored and co-authored approximately 300 research papers and has attracted over $£ 24$ million in research funding.

Prof. McGinnity is a fellow of the Institution of Engineering and Technology and a Chartered Engineer. 